TITLE:

\title{
Impulsive torque control of biped gait with power packets
}

$\operatorname{AUTHOR}(S)$ :

Mochiyama, Shiu; Hikihara, Takashi

CITATION:

Mochiyama, Shiu ... [et al]. Impulsive torque control of biped gait with power packets. Nonlinear Dynamics 2020, 102: 951-963

ISSUE DATE:

2020-10

URL:

http://hdl.handle.net/2433/259227

\section{RIGHT:}

This is a post-peer-review, pre-copyedit version of an article published in 'Nonlinear Dynamics'. The final authenticated version is available online at: https://doi.org/10.1007/s11071-020-05756-7.; The full-text file will be made open to the public on 20 June 2021 in accordance with publisher's 'Terms and Conditions for Self-Archiving'; この論文は出版社版で ありません。引用の際には出版社版をご確認ご利用ください。; This is not the published version. Please cite only the published version. 


\title{
Impulsive torque control of biped gait with power packets*
}

\author{
Shiu Mochiyama ${ }^{\dagger} \quad$ Takashi Hikihara \\ Kyoto University Katsura, Nishikyo, Kyoto, 615-8510 Japan \\ †s-mochiyama@dove.kuee.kyoto-u.ac.jp
}

\section{Abstract}

Many strategies for an actuated biped gait generation have been proposed based on the passive dynamic gait. Among them, this study focuses on an impulsive excitation at the toe-off instance. The strategy offers advantages in its experimental implementation; for example, it is not required to measure and control the trajectory of the legs all the time. However, there has been no study on a realistic design of the impulsive torque itself. In this paper, we propose an impulsive actuation method based on a power packet dispatching system. Power packet is a unit of electric power transfer in a pulse shape with information tags attached in voltage waveforms. According to the tag, power packets are transferred from sources to loads. On the basis of the power packetization, the torque input is configured as a result of a power packet supply to electric motors in a realistic setup. The proposed scheme controls the supply in a digitized way, that is, by changing the number of power packets supplied in a gait step. We confirm the successful gait generation with the power packets through numerical simulations.

Keywords: Power packet; Impulsive torque; Biped robot; Gait generation

\section{Nomenclature}

a Distance from the hip to the centroid of the leg.

$b \quad$ Distance from the ankle to the centroid of the leg.

$\boldsymbol{d}$ Initial state deviation from an equilibrium point.

$\boldsymbol{e}_{j} \quad$ Unit vector consisting of 6 elements.

Only the $j$-th element is 1 and others 0 .

$g \quad$ Gravitational acceleration.

$\mathrm{i}=\sqrt{-1}$. Imaginary unit.

$\boldsymbol{i}=\left[\begin{array}{ll}i_{1} & i_{2}\end{array}\right]^{\top}$.

$i_{j} \quad$ Current of motor $j$.

$l=a+b$. Length of each leg.

$m$ Mass of each leg.

$m_{\mathrm{H}} \quad$ Mass of the hip.

$n_{j} \quad$ Number of power packets supplied to motor $j$ in one gait step.

${ }^{*}$ This is a post-peer-review, pre-copyedit version of an article published in Nonlinear Dynamics. The final authenticated version is available online at: http://dx.doi.org/10.1007/s11071-02005756-7

\author{
$u_{j} \quad$ Output torque of motor $j$. \\ $\boldsymbol{v}=\left[\begin{array}{ll}v_{1} & v_{2}\end{array}\right]^{\top}$. \\ $v_{j} \quad$ Input voltage of motor $j$. \\ $\boldsymbol{x}=\left[\begin{array}{lll}\boldsymbol{\theta}^{\top} & \dot{\boldsymbol{\theta}}^{\top} & \boldsymbol{i}^{\top}\end{array}\right]^{\top}$. State point. \\ $\boldsymbol{x}_{0} \quad$ Initial state. \\ $\overline{\boldsymbol{x}}_{(j, k)}$ Equilibrium point of Poincaré map when \\ $\left(n_{1}, n_{2}\right)=(j, k)$. \\ $\mathcal{D} \mathcal{F}$ Linearized map of $\mathcal{F}$. \\ $\mathcal{F} \quad$ Poincaré map representing one gait step. \\ $K_{j} \quad$ Electromotive force constant of motor $j$. \\ $L_{j} \quad$ Inductance of the winding of motor $j$. \\ $R_{j} \quad$ Resistance of the winding of motor $j$. \\ $T$ Time duration of a power packet. \\ $V \quad$ Voltage of a power packet. \\ $\alpha=\left(\theta_{1}-\theta_{2}\right) / 2$. Hip joint angle divided by 2 . \\ $\gamma \quad$ Slope angle. \\ $\varepsilon \quad$ Scalar perturbation for eigenvalue computations. \\ $\boldsymbol{\theta}=\left[\begin{array}{ll}\theta_{1} & \theta_{2}\end{array}\right]^{\top}$. Generalized coordinates. \\ $\dot{\boldsymbol{\theta}}$ Time derivative of $\boldsymbol{\theta}$. \\ $\ddot{\boldsymbol{\theta}}$ Time derivative of $\dot{\boldsymbol{\theta}}$. \\ $\theta_{1} \quad$ Angle of stance leg. \\ $\theta_{2} \quad$ Angle of swing leg. \\ $\theta_{j}^{-} \quad$ Value of $\theta_{j}$ right before an impact. \\ $\theta_{j}^{+} \quad$ Value of $\theta_{j}$ right after an impact. \\ $\dot{\boldsymbol{\theta}}^{-} \quad$ Value of $\dot{\boldsymbol{\theta}}$ right before an impact. \\ $\dot{\boldsymbol{\theta}}^{+} \quad$ Value of $\dot{\boldsymbol{\theta}}$ right after an impact. \\ $\kappa_{j} \quad$ Gear ratio of motor $j$. \\ $\boldsymbol{\lambda}$ Eigenvalues of an equilibrium point. \\ $\tau \quad$ Generalized force. \\ $\omega_{j} \quad$ Angular velocity of the joint where motor $j$ is \\ mounted.
}

\section{Introduction}

McGeer [1] showed that a simple biped robot exhibits a passive dynamic walking on a gentle downhill. The passive walker exploits the gravity to generate a stable gait without external actuation. Following the study, much effort has been devoted to the further understanding of the underlying mechanisms and rich properties such as bifurcation and chaos [2-4]. An important tool for their analysis is Poincaré map [2,5], whose fixed point cor- 
responds to a periodic gait. Eigenvalue analysis based on the linearization of the map provides the information of stability of the gait. Stability apparently plays an important role in the practical use of the gait; however, due to the complexity of the biped dynamics, it is not an easy task to derive the map. Much effort has been devoted to the constitution method of the map both numerically and analytically $[2,3,6]$. Besides the local property, as a global analysis, a basin of attraction of a gait has also been actively investigated until recently. There have been not only numerical methods to obtain the basin [7], but also analyses of the mechanism how its complex shape appears [8-10].

Along with the analytic studies, another research direction is the utilization of the dynamics of the passive walker for an actuated gait. The work of Goswami et al. [11] is known as the first proposal of the active gait control. Focusing on the exchange of kinetic and potential energy that occurs every step in the passive gait, they proposed an energy-tracking based strategy. Many research groups followed the study, and a variety of energy based strategies have been proposed until today: e.g. a virtual gravity based strategy by Asano et al. [12], an energy shaping based strategy by Spong et al. [13], Sinnet and Ames [14], de-León-Gómez et al. [15], and Yeatman et al. [16]. Apart from the energy based ones, there have recently been many proposals of active control strategies from various perspectives, including a bio-mimetic approach by Fu et al. [17, 18], an OGY-based feedback control by Gritli et al. [19,20], and impulsive input strategies by Kuo [21], Moon et al. [22], and Mochiyama and Hikihara [23].

In the literature reviewed above, a common and essential point is to utilize the intrinsic dynamics of the passive gait. The purely passive gait is ultimately energy efficient since it does not require any external energy injection except for its own potential energy. Thus, even in an actuated gait, it would be effective to keep the intrinsic dynamics as much as possible, instead of overwriting it with a high-gain input [19]. The standpoint is also taken over in the gait control introduced in this paper.

Among the various strategies, we focus on an impulsive excitation [21-23], where an impulsive torque is applied only at the toe-off instance. The point is that the natural dynamics of the mechanical configuration is kept in almost all of the gait period. That is, it is not required to control the swing-leg trajectory. This becomes an advantage in an experimental implementation; the trajectory control requires a measurement of the state variables and a regulation of the input in a precise and real-time manner.

In spite of its high affinity for the real applications, however, few studies have addressed a design problem of the impulsive torque. Obviously, it is impossible to produce a pure impulse in a robot. To bring it to the stage of applications, it is required to discuss a realistic

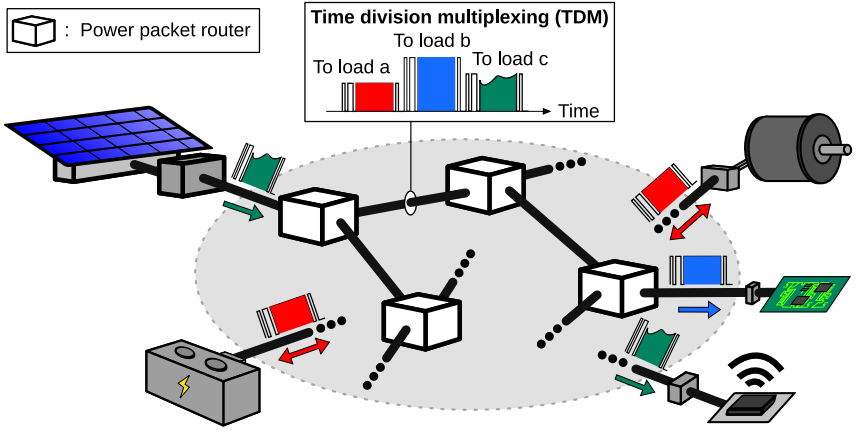

Figure 1: Power packet dispatching system.

impulsive actuation.

This paper is devoted to the impulsive torque control by means of power packet. Power packet dispatching system has been proposed for smart management of electric power flows [24-28]. Figure 1 shows an example of the power packet dispatching system. In the proposal, a power supply for a load is represented by an intermittent sequence of discrete-valued inputs called power packets. A power packet is a unit of electric power transfer in a pulse shape with information tags attached in voltage waveforms. According to the tag, power packets are transferred from sources to loads. The system offers many advantages especially in systems such as a mobile robot and an electric vehicle: the smooth inclusion of power sources of time-varying profiles, the reduction of wires and power converters, and so on [25]. In addition, the quantization of power enables a computation of power flow in a completely digitized way. Here arises the possibility of using the packetized power for the impulsive actuation in the biped robot.

Recently, a load control method based on the packetized power has been developed $[29,30]$. In addition, the concept has been experimentally proved to be available for the power level of industrial applications [Mochiyama, et al., under review]. The realization of the concept largely owes to the emerging semiconductor power devices such as $\mathrm{SiC}$ [31]. Based on the developed hardware, we consider the impulsive actuation with power packets.

In this paper, we first define the numerical models of the robot and the impulsive excitation with power packets. Then, the impulsive torque control method is developed on the basis of the models. The feasibility of the gait generation with the developed method is confirmed through numerical simulations. Lastly, to support the feasibility of the proposed control in the real applications, we provide an analysis of the generated gait from the perspectives of stability and attraction. The stability is confirmed through numerical method called perturbation technique $[2,20]$. The basin of attraction is also obtained numerically through a simple exhaustive search in a certain area of the state space. 


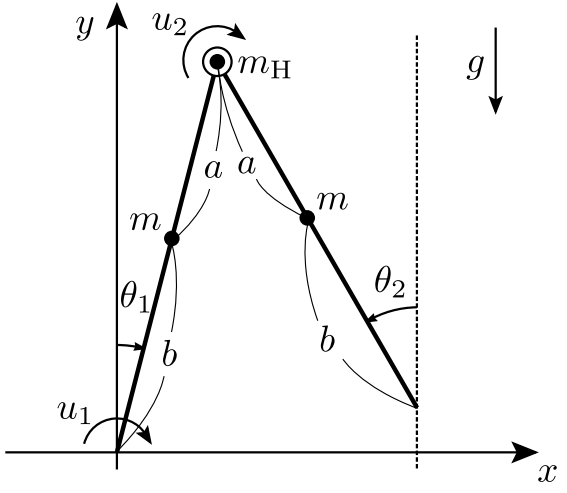

Figure 2: Compass model of the biped robot.

\section{Compass Model of Biped Robot}

\subsection{Configuration}

Here is introduced a compass model of a biped robot referring to [2]. Figure 2 shows the physical configuration of the robot. The robot walks on a flat horizontal ground. The generalized coordinates are defined by the angles of the legs, $\boldsymbol{\theta}=\left[\begin{array}{ll}\theta_{1} & \theta_{2}\end{array}\right]^{\top}$. The robot is actuated by the motors placed at the ankle and the hip joint. The input torques, $u_{1}$ and $u_{2}$, are exerted on the ankle and the hip joint, respectively.

Now note that there are also studies on an underactuated biped robot (e.g. $[19,32,33])$, as well as a fully actuated one (e.g. $[12,13,22])$. For example, the robot discussed in [32] exhibits a stable gait only with the ankle joint powered. Still, we selected the fully actuated model because we have confirmed in the previous study [23] that the fully actuated model exhibits a variety of gaits (in terms of gait speed and period) by controlling the torque distribution between the ankle and hip actuators. This feature is expected to be useful for more flexible control of the gait by power packets.

A gait of the model can be divided into two phases: (a) swing phase, and (b) impact phase. In the phase (a), the swing leg moves in the air, while the stance leg is fixed on the ground. The impulsive input is applied at the beginning of this phase. Then, the gait moves onto the phase (b) when the swing leg collides with the ground. In this phase, the swing and stance legs switch their roles. After the phase (b), the phase (a) starts again. The numerical models for the phases are shown in what follows.

\section{$2.2 \quad$ Numerical Models}

The equations of motion of the robot in the phase (a) can be derived through Lagrangian formulation [34]. The Lagrangian of the robot is

$$
\mathcal{L}(\boldsymbol{\theta}, \dot{\boldsymbol{\theta}})=K(\boldsymbol{\theta}, \dot{\boldsymbol{\theta}})-P(\boldsymbol{\theta}),
$$

where

$$
\begin{aligned}
K(\boldsymbol{\theta}, \dot{\boldsymbol{\theta}})= & \frac{1}{2} \dot{\boldsymbol{\theta}}^{\top} \boldsymbol{M}(\boldsymbol{\theta}) \dot{\boldsymbol{\theta}}, \\
P(\boldsymbol{\theta})= & \left\{m_{\mathrm{H}} l \sin \theta_{1}+m b \cos \theta_{1}\right. \\
& \left.+m\left(l \cos \theta_{1}-a \cos \theta_{2}\right)\right\} g, \\
\boldsymbol{M}(\boldsymbol{\theta})= & {\left[\begin{array}{cc}
\left(m_{\mathrm{H}}+m\right) l^{2}+m b^{2} & -m a l \cos 2 \alpha \\
-m a l \cos 2 \alpha & m a^{2}
\end{array}\right] . }
\end{aligned}
$$

As the generalized force related to the generalized coordinates is

$$
\boldsymbol{\tau}=\boldsymbol{S} \boldsymbol{u}=\left[\begin{array}{cc}
1 & 1 \\
0 & -1
\end{array}\right]\left[\begin{array}{l}
u_{1} \\
u_{2}
\end{array}\right],
$$

the equations of motion of the robot in the phase (a) is expressed by

$$
\frac{\mathrm{d}}{\mathrm{d} t} \frac{\partial \mathcal{L}}{\partial \dot{\boldsymbol{\theta}}}-\frac{\partial \mathcal{L}}{\partial \boldsymbol{\theta}}=\tau .
$$

Substituting Eq. (1) into Eq. (6), we have

$$
\boldsymbol{M}(\boldsymbol{\theta}) \ddot{\boldsymbol{\theta}}+\boldsymbol{C}(\boldsymbol{\theta}, \dot{\boldsymbol{\theta}}) \dot{\boldsymbol{\theta}}+\boldsymbol{g}(\boldsymbol{\theta})=\boldsymbol{\tau},
$$

where

$$
\begin{aligned}
\boldsymbol{C}(\boldsymbol{\theta}, \dot{\boldsymbol{\theta}}) & =\left[\begin{array}{cc}
0 & -m a l \dot{\dot{\theta}_{2}} \sin 2 \alpha \\
m a l \dot{\theta_{1}} \sin 2 \alpha & 0
\end{array}\right], \\
\boldsymbol{g}(\boldsymbol{\theta}) & =\left[\begin{array}{c}
-\left(m_{\mathrm{H}} l+m b+m l\right) \sin \theta_{1} \\
m a \sin \theta_{2}
\end{array}\right] g .
\end{aligned}
$$

The input torque is supplied from the actuators. We consider a linear model of a brushed dc motor

$$
\left\{\begin{array}{l}
L_{j} \dot{i}_{j}=-R i_{j}-\kappa_{j} K_{j} \omega_{j}+v_{j}, \\
u_{j}=\kappa_{j} K_{j} i_{j}
\end{array},\right.
$$

where $\omega_{j}$ represents the angular velocity of the joint where motor $j$ is mounted: $\omega_{1}=\dot{\theta}_{1}$ and $\omega_{2}=\dot{\theta}_{1}-\dot{\theta}_{2}$. In other words, $\kappa \omega_{j}$ represents the angular velocity of the shaft of motor $j$. The input of Eq. (10) is set as the voltage $v$, which is realized by the input of power packets. The design of the power packets will be discussed later.

The phase (a) ends by the impact of the swing leg on the ground. When the ground is a slope of $\gamma \mathrm{rad}$, the equation

$$
\theta_{1}^{-}+\theta_{2}^{-}=2 \gamma
$$

holds at the impact, where $\theta_{1}^{-}$and $\theta_{2}^{-}$represent $\theta_{1}$ and $\theta_{2}$ right before the impact. In other words, the transition of the legs occurs when the state variables transversely crosses a hyperplane

$$
\Sigma: \theta_{1}+\theta_{2}-2 \gamma=0
$$

Throughout this paper, we consider a flat ground and $\gamma$ is fixed at zero. We assume that the impact of a swing leg can be modeled as an instantaneous and inelastic collision. Let $\dot{\boldsymbol{\theta}}^{-}$and $\dot{\boldsymbol{\theta}}^{+}$be angular velocities of right before and after the impact. Then the law of conservation of angular momentum leads to

$$
\boldsymbol{Q}^{+}(\alpha) \dot{\boldsymbol{\theta}}^{+}=\boldsymbol{Q}^{-}(\alpha) \dot{\boldsymbol{\theta}}^{-},
$$




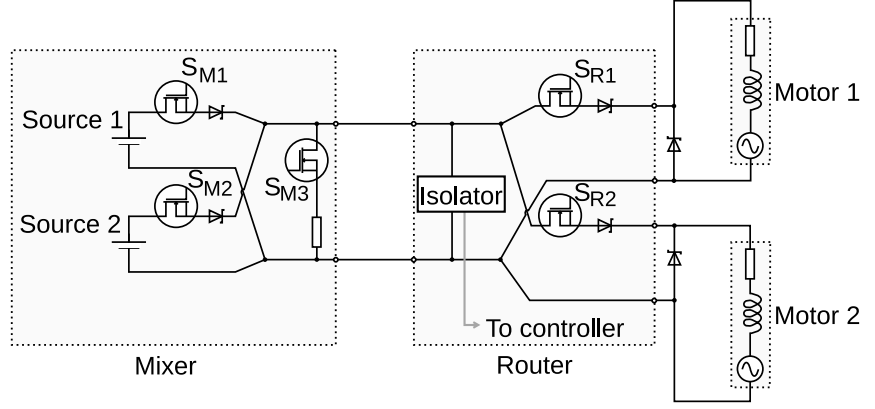

Figure 3: Power packet dispatching circuit.

where

$$
\begin{aligned}
\boldsymbol{Q}^{+}(\alpha) & =\left[\begin{array}{ll}
q_{11}^{+} & q_{12}^{+} \\
q_{21}^{+} & q_{22}^{+}
\end{array}\right], \\
q_{11}^{+} & =m_{\mathrm{H}} l^{2}+m b^{2}+m l(l-a \cos 2 \alpha), \\
q_{12}^{+} & =m a(a-l \cos 2 \alpha), \\
q_{21}^{+} & =-m a l \cos 2 \alpha, \\
q_{22}^{+} & =m a^{2}, \\
\boldsymbol{Q}^{-}(\alpha) & =\left[\begin{array}{ll}
q_{11}^{-} & q_{12}^{-} \\
q_{21}^{-} & q_{22}^{-}
\end{array}\right], \\
q_{11}^{-} & =\left(m_{\mathrm{H}} l^{2}+2 m b l\right) \cos 2 \alpha-m a b, \\
q_{12}^{-} & =q_{21}^{-}=-m a b, \\
q_{22}^{-} & =0 .
\end{aligned}
$$

Along with the jump of the angular velocity, the exchange between the swing and stance legs occurs at the impact. The switch is expressed as

$$
\boldsymbol{\theta}^{+}=\left[\begin{array}{ll}
0 & 1 \\
1 & 0
\end{array}\right] \boldsymbol{\theta}^{-}
$$

\section{Impulsive torque control with power packets}

\subsection{Dispatching system}

Figure 3 shows the power packet dispatching circuit. The circuit can be seen as a minimal setup of the general dispatching network shown in Fig. 1. The source $j$ supplies power to motor $j$. The torque $u_{j}$ is generated by the motor $j$. Note that the two motors placed at each ankle are unified as "motor 2." This simplification is based on the fact that the two ankles are never actuated at the same time.

The mixer generates a sequence of power packets with switches $\mathrm{SW}_{\mathrm{M} 1}, \mathrm{SW}_{\mathrm{M} 2}$, and $\mathrm{SW}_{\mathrm{M} 3}$. To output "high" logic or power, one of the serial-connected switches, $\mathrm{SW}_{\mathrm{M} 1}$ or $\mathrm{SW}_{\mathrm{M} 2}$, is turned on. Other switches are kept off to avoid a short circuit. To output "low" logic, the parallel-connected switch $\mathrm{SW}_{\mathrm{M} 3}$ is turned on, while $\mathrm{SW}_{\mathrm{M} 1}$ and $\mathrm{SW}_{\mathrm{M} 2}$ are kept off. The information tags are read by the router's controller through the isolator module. When the router finishes reading the header of a power packet, one of the switches $\mathrm{SW}_{\mathrm{R} 1}$ or $\mathrm{SW}_{\mathrm{R} 2}$ is turned on to supply the power packet to the corresponding motor. After detecting the end of the footer, the router turns off all the switches.

The actuators are supplied by power packets at the beginning of each step. The voltage and the time duration of a power packet are denoted by $V$ and $T$, respectively. The values are kept constant; that is, the amount of power is controlled by the number of power packets. We denote the number of power packets supplied to motor $j$ in one gait step by $n_{j}(j=1,2)$. Here power packets are supplied to each motor alternately in the time-division multiplexing (TDM) manner [24, 25]. The supply is then expressed as

$$
\boldsymbol{v}=\left\{\begin{array}{l}
{\left[\begin{array}{l}
V \\
0
\end{array}\right] \quad \text { if }\left\{\begin{array}{l}
(2 n-2) T \leq t<(2 n-1) T \\
n=1,2, \ldots, n_{1}
\end{array}\right.} \\
{\left[\begin{array}{l}
0 \\
V
\end{array}\right] \quad \text { if }\left\{\begin{array}{l}
(2 n-1) T \leq t<2 n T \\
n=1,2, \ldots, n_{2}
\end{array}\right.} \\
{\left[\begin{array}{l}
0 \\
0
\end{array}\right] \quad \text { otherwise }}
\end{array}\right.
$$

where the onset of the time $t$ coincides with the beginning of a step.

Figure 4 shows an example of the TDM input in the case of $\left(n_{1}, n_{2}\right)=(3,2)$. The top graph corresponds to the first element of $\boldsymbol{v}$, i.e. the supply to motor 1 , and the bottom graph to the second, i.e. the supply to motor 2. The supply to each motor is composed of multiple intermittent excitations. The two motors become active repeatedly and alternately in one gait step, until the number of activations reaches 3 and 2, respectively. The activation of motor 1 (motor 2) corresponds to the first (second) case of Eq. (24). After that, the two actuators are kept inactive until the beginning of the next gait step (until the next impact), which corresponds to the third case of Eq. (24). Here the time duration of each excitation, $T$, is much shorter than the gait period and the mechanical time constant, and there is no supply except for at the very beginning of the gait period. In these ways, the power-packet based controller takes over the essential feature from the previous proposals of the purely impulsive gait control. That is, it preserves the natural dynamics of the passive gait in almost all all of the gait period. In this sense, we call the controller as impulsive.

The TDM transfer is one of the key concepts in the power packet dispatching system [24,25]. The distinction in time domain enables the power system to identify the flow of each power packet in the router network. Obviously, this is not achieved in the common power system where power from/to different source/destination is mixed together. In addition, the system accommodates 


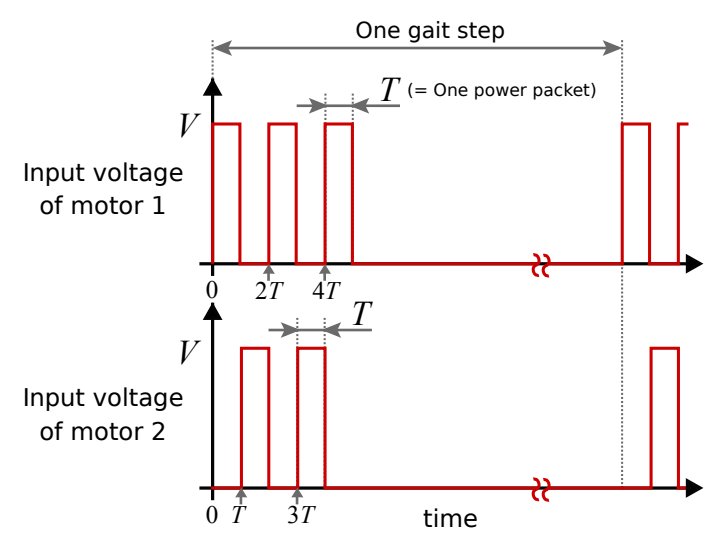

Figure 4: Example of the TDM input in the case of $\left(n_{1}, n_{2}\right)=(3,2)$.

the difference of voltage levels on a shared power line. These features contribute to the reduction of the wires and converters used in the conventional power system. Their reduction contributes to less weight and volume, i.e. to better maneuverability and higher energy efficiency. Now it should be noted that the efficiency above indicates that of the whole system including the power distribution system. Besides the high efficiency of the impulsive gait control scheme itself, the introduction of the power packet dispatching system is expected to enhance the efficiency of the whole system by serving as an interface between the control scheme and the physical system including its power system.

The proposed method determines the number of power packets in a feedforward manner. This is to remove the requirement for a precise measurement of the state variable to design the input torque. This is advantageous for the applications since the real-time measurement and computation are not required. The simple strategy is also better suited for the concept of exploiting the natural dynamics of the structure as much as possible. Note that the stability of the generated gait, which is confirmed later in Section 4, supports the feasibility of the feedforward method.

\subsection{Setup of power packet}

Now we determine the specific setup of the power packet supply. To keep the feasibility in the process of the torque generation, we consider the following requirements. First, (R1) the maximum voltage and current of a power packet supply are set at the level which is adopted in a popular robotics application. Second, (R2) the length of a unit pulse (power packet) is set within a reasonable range. In (R2), the pulse length is required to be sufficiently shorter than the step period of the robot; otherwise it is no longer an impulsive excitation. At the same time, it cannot be too short to realize the flow of current in the switching circuit.

Now, setting a concrete configuration of the robot
Table 1: Parameters of the biped robot.

\begin{tabular}{ccc}
\hline Parameter & Unit & Value \\
\hline Mass of hip: $m_{\mathrm{H}}$ & $\mathrm{kg}$ & 10.0 \\
Mass of leg: $m$ & $\mathrm{~kg}$ & 5.0 \\
Distance from hip to centroid: $a$ & $\mathrm{~m}$ & 0.50 \\
Distance from ankle to centroid: $b$ & $\mathrm{~m}$ & 0.50 \\
\hline
\end{tabular}

Table 2: Parameters of the actuator (MAXON RE40 148877) and the attached gear.

\begin{tabular}{ccc}
\hline Parameter & Unit & Value \\
\hline Torque constant: $K$ & $\mathrm{~N} \mathrm{~m} \mathrm{~A}^{-1}$ & $6.03 \times 10^{-2}$ \\
Inductance: $L$ & $\mathrm{H}$ & $3.3 \times 10^{-4}$ \\
Resistance: $R$ & $\Omega$ & 1.13 \\
\hline Rated voltage & $\mathrm{V}$ & 48 \\
Max. cont. current & $\mathrm{A}$ & 3.17 \\
Starting current & $\mathrm{A}$ & 42.4 \\
Mass & $\mathrm{kg}$ & 0.48 \\
\hline Gear ratio: $\kappa$ & - & 16 \\
\hline
\end{tabular}

and the actuators, we confirm the existence of the setup which meets the requirements. Table 1 shows the values of the parameters related to the physical configuration of the robot. The parameters are referred to the previous study [2]. Dc brushed motors are adopted for the actuators of all the joints. Table 2 shows the values of the parameters related to the actuators (MAXON RE40 148877), taken from the website of the manufacturer [35]. The actuators are coupled to the ankles and the hip joint through gears. The gear ratio is set at $\kappa=16$ for all the actuators. The rated voltage is $48 \mathrm{~V}$, which is common for a robotic application. Its weight, $0.48 \mathrm{~kg}$, is also reasonable for loading onto the robot of the aforementioned physical configuration.

Based on the specifications of the motors, we determine the setups of power packet as follows. The voltage of power packets is set at the rated value, $V=48 \mathrm{~V}$. The packet length is set at $T=1.0 \times 10^{-2} \mathrm{~s}$. The length is expected to be much shorter than the step period of the robot, typically $0.7-1 \mathrm{~s}$, according to the previous studies using the similar model $[2,23]$. It is also sufficiently long compared with the electrical time constant of the motor. In fact, $T$ is about thirty times greater than the time constant of $L / R \sim 2.9 \times 10^{-4} \mathrm{~s}$. Thus it is expected that the current reaches its peak during each payload.

\section{Numerical simulation of gait generation}

For the numerical integration in the following simulations, the function "ode45" of MATLAB is used with the relative and absolute error tolerance parameters set at $1 \times 10^{-8}$ and $1 \times 10^{-10}$, respectively. The impact condition Eq. (12) is detected by the optional argument 
called "event" of the function.

\subsection{Results with $\left(n_{1}, n_{2}\right)=(6,4)$ and $(2,6)$}

This subsection shows an example of the successful gait generation with two specific setups of $\left(n_{1}, n_{2}\right)$. According to the literature on the purely impulsive gait generation [22,23], it is expected that a periodic gait is obtained under an appropriate selection of $\left(n_{1}, n_{2}\right)$. Here we introduce a discrete map $\mathcal{F}$ called a step-to-step map [3]. This is a Poincaré map of the state trajectory which is defined as a map from the state point right after an impact to the one right after the next impact. The state point is denoted by $\boldsymbol{x}=\left[\boldsymbol{\theta}^{\top}, \dot{\boldsymbol{\theta}}^{\top}, \boldsymbol{i}^{\top}\right]^{\top}$. Then a periodic gait corresponds to a fixed point of the map. To find combinations of $\left(n_{1}, n_{2}\right)$ and corresponding fixed point, we conduct some trial-and-error simulations in advance. In detail, for each $\left(n_{1}, n_{2}\right)$, we repeat simulations with initial states placed on lattice points in the state space and see if $\boldsymbol{x}=\mathcal{F}(\boldsymbol{x})$. As a result, we obtained the following two combinations, $\left\{\left(n_{1}, n_{2}\right)=(6,4), \overline{\boldsymbol{x}}_{(6,4)}\right\}$ and $\left\{\left(n_{1}, n_{2}\right)=(2,6), \overline{\boldsymbol{x}}_{(2,6)}\right\}$. The details of each gait are presented in the following.

First, we present the result of a gait simulation with $\left(n_{1}, n_{2}\right)=(6,4)$. Starting from the initial state

$$
\boldsymbol{x}_{0}=\overline{\boldsymbol{x}}_{(6,4)}=\left[\begin{array}{c}
-0.2209 \\
0.2209 \\
0.9670 \\
0.5488 \\
-1.023 \\
0.1978
\end{array}\right],
$$

the gait continues at least 100 steps without falling down. Figure 5 shows the trajectories of angle, angular velocity, current, and torque for the first 8 seconds of the gait. Figures 5 (a) and (b) show the trajectories of angle and angular velocity. The discontinuous changes in angle and velocity indicate the impacts of the swing leg to the ground. The trajectories between each impact exhibit the same appearance. Figures 5 (c) and (d) show the trajectories of current. The current pulses are generated at the beginning of each step. The magnitude of each pulse is kept constant.The periodic trajectories of angle, angular velocity, and current indicate the appearance of a periodic gait. That is, in the steady state, each step begins with the same state point, namely $\boldsymbol{x}_{0}$. Figures 5 (e) and (f) show the enlarged view of the current trajectories. The current pulses are generated alternately at motors 1 and 2, which shows that the power packets are transferred in the TDM manner. The current trajectory in each power packet reaches its peak value, as is expected in the setup. Figures $5(\mathrm{~g})$ and $(\mathrm{h})$ show the trajectories of torque. The impulsive torque is generated in accordance with the current pulses. The trajectories of torque almost coincide with the current, since the torque is proportional to the current and its factor is about 1 (a)
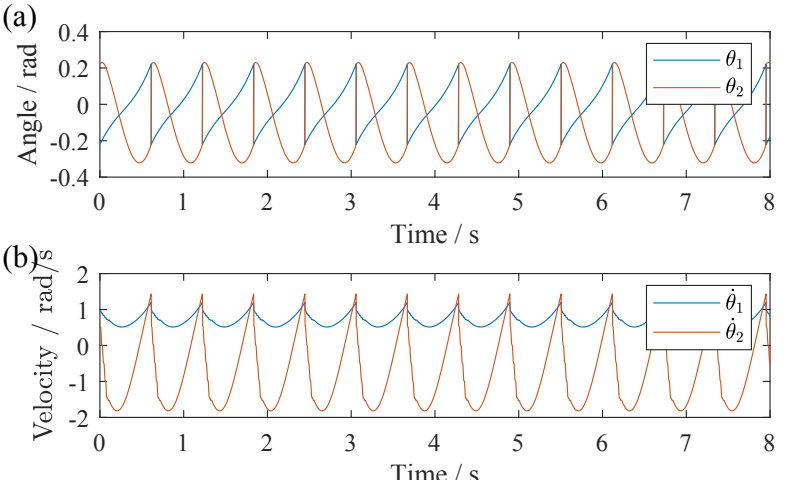

(c)

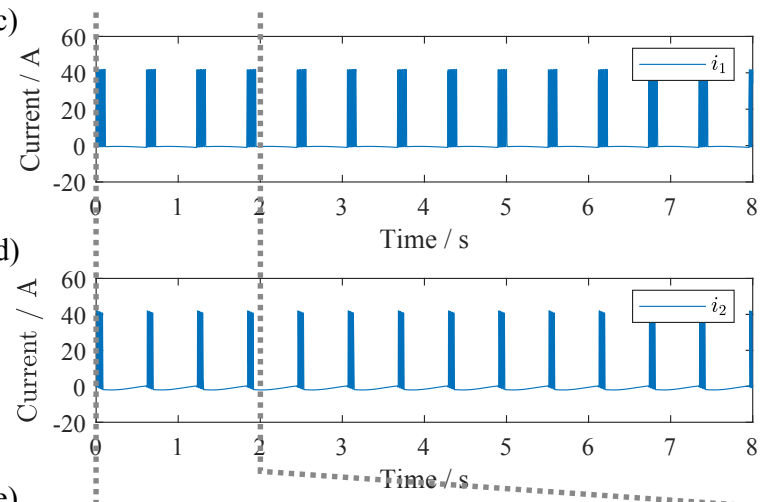

(e)

(f)

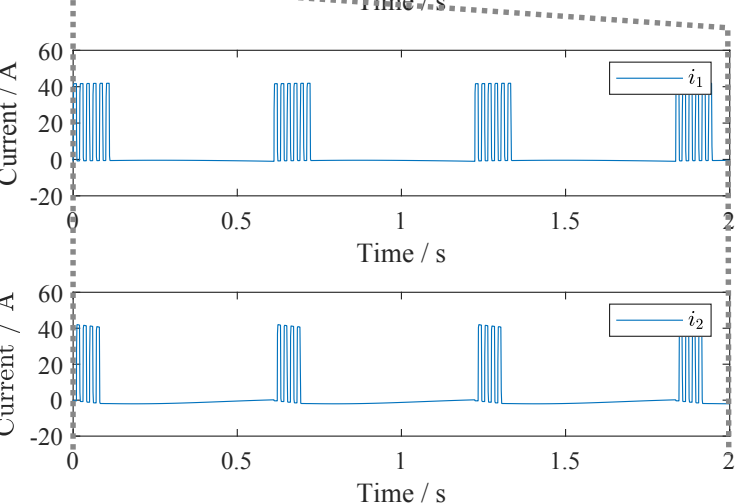

(g)
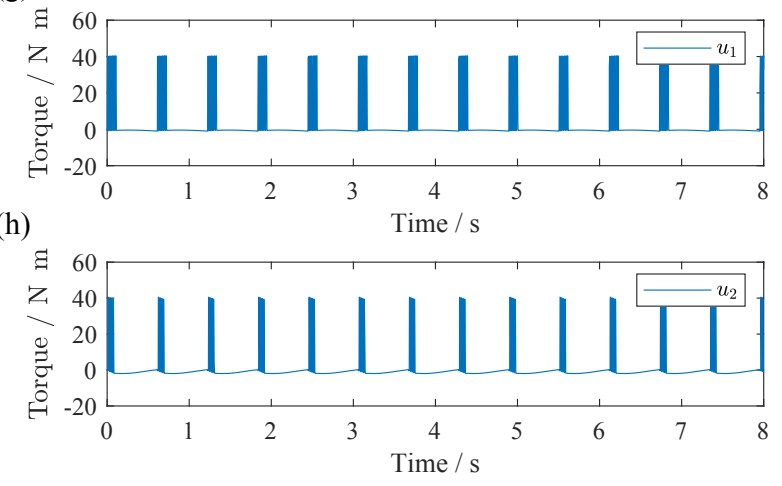

Figure 5: Results of the simulation with $\left(n_{1}, n_{2}\right)=(6,4)$. (a) Angle trajectories of both legs. (b) Angular velocity trajectories of both legs. (c) Current trajectory of motor 1. (d) Current trajectory of motor 2. (e) Enlarged view of current trajectory of motor 1. (f) Enlarged view of current trajectory of motor 2. (g) Torque trajectory of motor 1. (h) Torque trajectory of motor 2. 
$\left(\kappa_{j} \cdot K_{j} \sim 1\right)$. To sum up the results above, we conclude that the generation of a periodic gait can be realized with impulsive actuation by power packets.

Then we move on to another setup: $\left(n_{1}, n_{2}\right)=(2,6)$. Starting from the initial state

$$
\boldsymbol{x}_{0}=\overline{\boldsymbol{x}}_{(2,6)}=\left[\begin{array}{c}
-0.1835 \\
0.1835 \\
0.8058 \\
0.3978 \\
-0.9439 \\
1.326
\end{array}\right],
$$

the gait also continues at least 100 steps without falling down. Figure 6 shows the result with $\left(n_{1}, n_{2}\right)=(2,6)$. Figures $6(\mathrm{a})$ - (d) show that the state exhibits a periodic change in every step, as is observed for $\left(n_{1}, n_{2}\right)=(6,4)$. The comparison of Figs. 5 (e) and (f) and Figs. 6 (e) and (f) indicates the change of the number of input power packets according to the setup of $\left(n_{1}, n_{2}\right)$.

Now, in the successful gaits, some differences are found depending on the value of $\left(n_{1}, n_{2}\right)$. For example, the amplitudes of the angle trajectories and the gait period are different between the results with $\left(n_{1}, n_{2}\right)=(6,4)$ and with $\left(n_{1}, n_{2}\right)=(2,6)$. Compared with the result with $\left(n_{1}, n_{2}\right)=(6,4)$, both the amplitudes of the angle trajectories and the gait period are increased. This result suggests the possibility of gait control by changing the number of power packets in a feedforward manner.

\subsection{Property of attraction}

The generated gait possesses a property of attraction under small deviation of the initial value. To confirm the property, we first analyze stability of the periodic gait. The local stability of the periodic gait can be determined through a stability analysis of the corresponding fixed point of the Poincaré map $\mathcal{F}$ [5]. Let the linearized map of $\mathcal{F}$ be denoted by $\mathcal{D F}$. According to Hartman-Grobman's theorem, if $\mathcal{D} \mathcal{F}(\overline{\boldsymbol{x}})$ has no zero or purely imaginary eigenvalues, the local behavior in a neighborhood of $\overline{\boldsymbol{x}}$ is identical both in the original and the linearized cases [36]. There have been several methods to constitute the linearized map, both analytical approaches $[6,37]$ and numerical ones $[2,3,20]$. We adopt what is called the perturbation technique based on numerical integration, following [20]. The procedure is summarized in Algorithm 1. Each column of $\mathcal{D F}$ is derived through a calculation of how a small perturbation grows after one step of the gait. Note that it is required to set the value of $\varepsilon$ appropriately; too large or small value of $\varepsilon$ can lead to a significant error of the obtained map [20]. We thus repeat the procedure with different $\varepsilon$ to determine the best value, i.e. the value around which a small change of $\varepsilon$ does not affect the result of the calculation. It is also worth noting that the perturbation technique is not efficient especially in the (a)

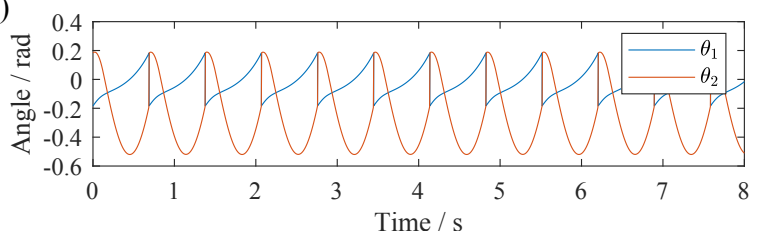

(b)

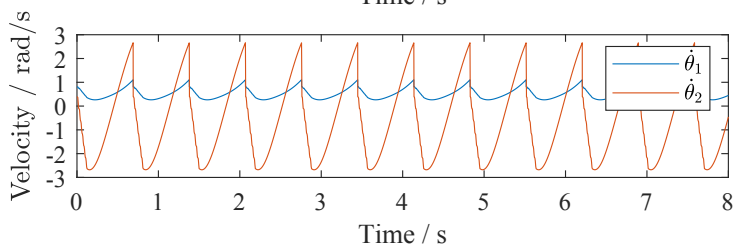

(c)

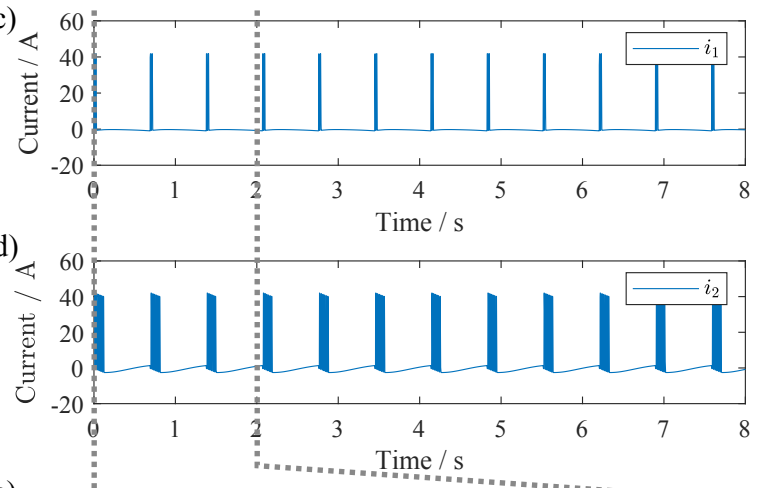

(e)

(f)

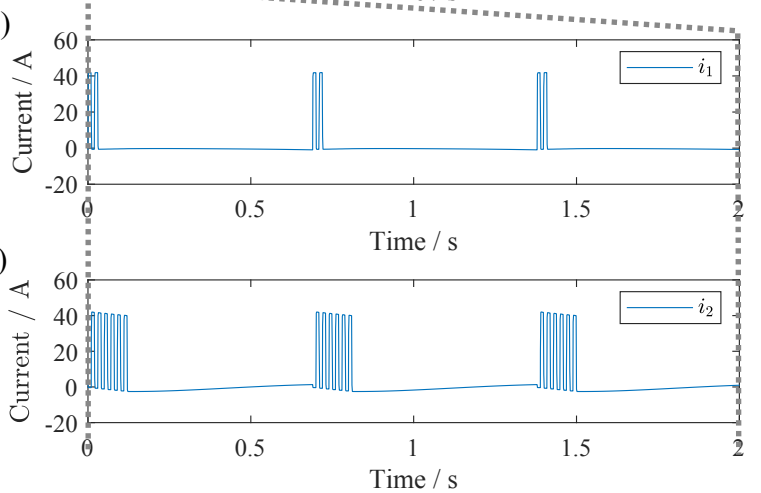

(g)

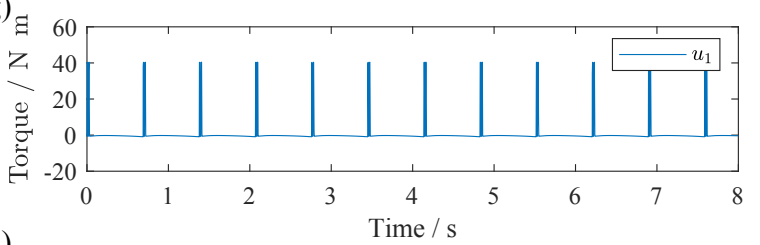

(h) ्ㅏ

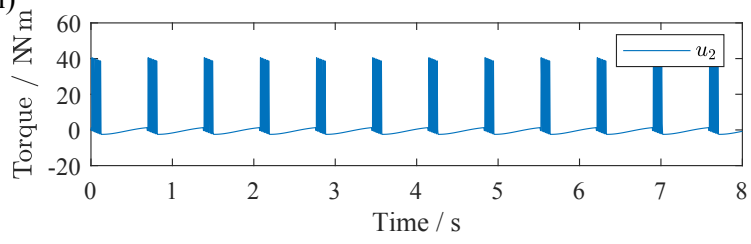

Figure 6: Results of the simulation with $\left(n_{1}, n_{2}\right)=(2,6)$. (a) Angle trajectories of both legs. (b) Angular velocity trajectories of both legs. (c) Current trajectory of motor 1. (d) Current trajectory of motor 2. (e) Enlarged view of current trajectory of motor 1. (f) Enlarged view of current trajectory of motor 2. (g) Torque trajectory of motor 1. (h) Torque trajectory of motor 2. 


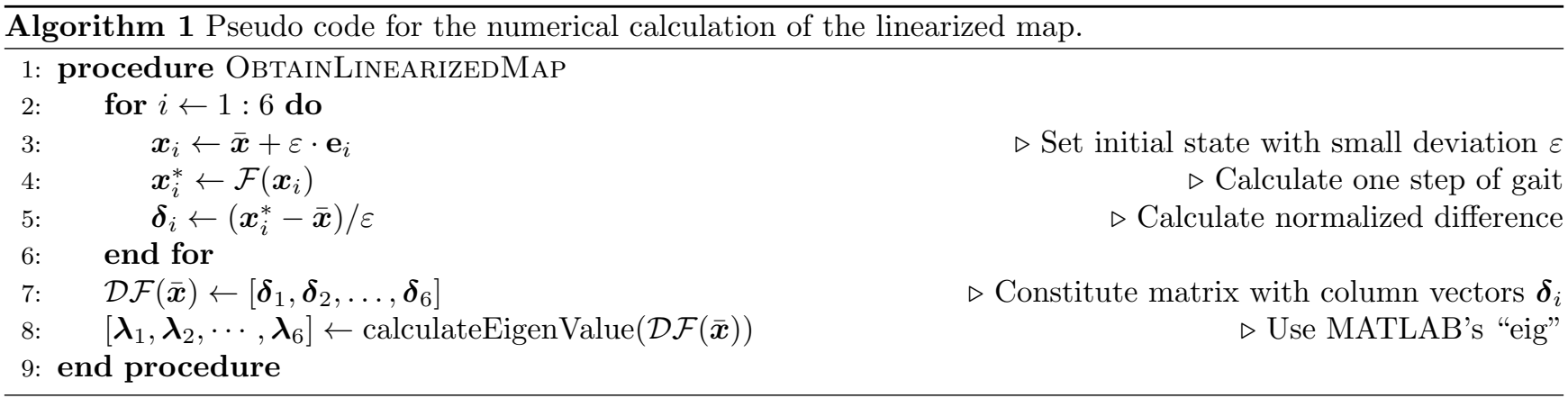

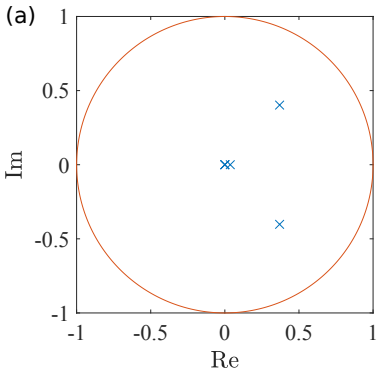

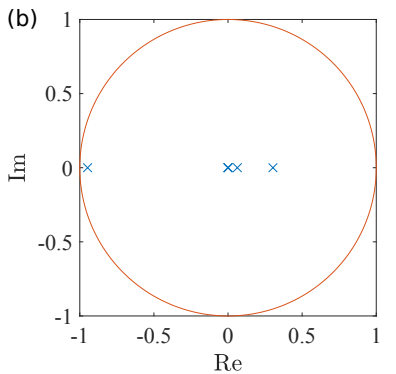

Figure 7: Eigenvalues on the complex plane. The symbols " $x$ " denote the eigenvalues. Note that some symbols around the origin are overlapped because of their close values.

analysis of unstable gait [20]. Although this issue does not apply to our case, where only stable gaits are considered, it should be noted for further investigations e.g. a bifurcation analysis.

With the procedure above, the eigenvalues $\boldsymbol{\lambda}_{(6,4)}$ and $\boldsymbol{\lambda}_{(2,6)}$ are obtained as follows:

$$
\begin{gathered}
\boldsymbol{\lambda}_{(6,4)}=\left[\begin{array}{c}
0.3694+0.4022 \mathrm{i} \\
0.3694-0.4022 \mathrm{i} \\
3.665 \times 10^{-2} \\
1.303 \times 10^{-8}+8.521 \times 10^{-8} \mathrm{i} \\
1.303 \times 10^{-8}-8.521 \times 10^{-8} \mathrm{i} \\
-3.142 \times 10^{-5}
\end{array}\right], \\
\boldsymbol{\lambda}_{(2,6)}=\left[\begin{array}{c}
-0.9475 \\
0.3030 \\
6.300 \times 10^{-2} \\
-4.867 \times 10^{-6}+4.252 \times 10^{-6} \mathrm{i} \\
-4.867 \times 10^{-6}-4.252 \times 10^{-6} \mathrm{i} \\
2.4167 \times 10^{-5}
\end{array}\right] .
\end{gathered}
$$

Figure 7 shows the eigenvalues plotted on the complex plane. All the eigenvalues are placed within a unit circle, so the one-period gaits obtained above are locally stable.

Besides the local stability, global attraction is also an important property from the practical point of view. For a purely passive gait, the basin of attraction of $\overline{\boldsymbol{x}}$ has been obtained through numerical integration and the mechanism of how it is formed in the state space has been investigated [7-10]. Furthermore, for an actuated gait with an impulsive excitation, the basin of attraction has been obtained and it appears similar to the purely passive case [22]. On the basis of the fact, it is expected that a similar basin can be obtained for the gait control considered in this paper. Although the detailed analysis of the basin itself is beyond the scope of this paper, it is helpful for the discussion on the feasibility of the proposed control to investigate a rough outline of the basin. Thus, in what follows, we compute the basin in the case of $\left(n_{1}, n_{2}\right)=(6,4)$ through numerical integration.

The basin lies in the five dimensional space $\left(\theta_{1}\right.$, $\left.\dot{\theta}_{1}, \dot{\theta}_{2}, i_{1}, i_{2}\right)^{1}$, which means the direct visualization is difficult. We thus show the basin projected to the three dimensional space $\left(\theta_{1}, \dot{\theta}_{1}, \dot{\theta}_{2}\right)$ with $\left(i_{1}, i_{2}\right)$ fixed at the equilibrium value. The computation is conducted with a set of $50 \times 50$ initial state points placed on a planer lattice. The lattice is set on the $\theta_{1}-\dot{\theta}_{1}$ plane with $\dot{\theta}_{2}$ fixed at a specific value. By changing the value of $\dot{\theta}_{2}$, we obtain sliced views of the $3 \mathrm{~d}$ basin. Figure 8 (a) shows the set of initial state points used for the computation. The $3 \mathrm{~d}$ grid consists of 50,50, and 9 points along the axes of $\theta_{1}$, $\dot{\theta}_{1}$, and $\dot{\theta}_{2}$, respectively. The center of the grid coincides with the equilibrium point. An initial point is regarded to be a part of the basin if the gait converges to the equilibrium point in 50 gait steps. Now the omission of $\left(i_{1}, i_{2}\right)$ can be justified by the fact that the initial value of $\left(i_{1}, i_{2}\right)$ is much smaller than the rated current of the motors. In other words, the effect of current deviations that can appear in a practical situation is considered to be extremely small. This point is confirmed through numerical simulations later.

Figures 8 (b)-(f) show the computed basin. On each layer, the basin has a narrow band shape with the branched point moving toward the equilibrium as $\dot{\theta}_{2}$ decreases. The overall appearance seems similar to the previous results in a purely passive gait $[8,9]$ and in an actuated gait with pure impulses [22]. Note that the setup of the grid does not include the whole basin. In other words, the basin might continue to the state space outside the grid.

Now, in order to show an example of the attracting

\footnotetext{
${ }^{1}$ As we consider the point right after the impact, $\theta_{2}$ is dependent on $\theta_{1}$ under the relationship of Eq. 12 .
} 

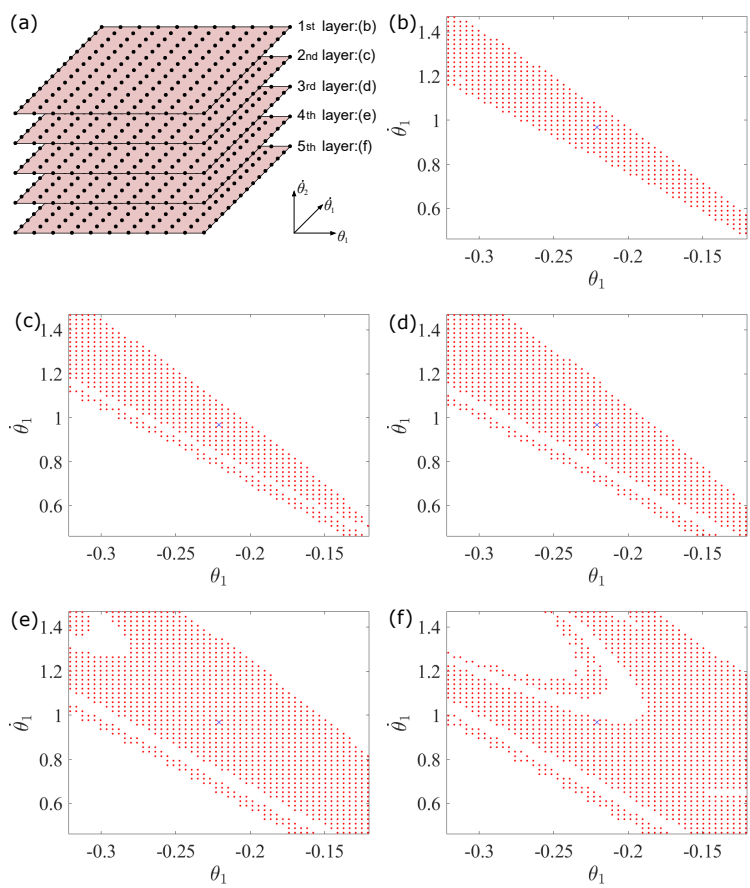

Figure 8: Basin of attraction. (a) Set of initial state points used for the computation. (b) - (f) Obtained basin of attraction on each sliced layer indicated in (a).

property, we examine the gait of $\left(n_{1}, n_{2}\right)=(6,4)$ with an initial state including the following sets of small deviation:

$$
\begin{aligned}
& \boldsymbol{d}_{1}=\left[\begin{array}{llllll}
0.030, & 0.0, & 0.0, & 0.0, & 0.0, & 0.0
\end{array}\right]^{\top}, \\
& \boldsymbol{d}_{2}=\left[\begin{array}{llllll}
0.0, & 0.0, & -0.14, & -0.4, & 0.0, & 0.0
\end{array}\right]^{\top}, \\
& \boldsymbol{d}_{3}=\left[\begin{array}{llllll}
0.0, & 0.0, & 0.0, & 1.2, & 0.0, & 0.0
\end{array}\right]^{\top}, \\
& \boldsymbol{d}_{4}=\left[\begin{array}{llllll}
0.0, & 0.0, & 0.0, & 0.0, & 0.6, & 1.2
\end{array}\right]^{\top} .
\end{aligned}
$$

The initial state is defined using the deviation as

$$
\boldsymbol{x}_{d_{j}}=\boldsymbol{x}_{0}+\boldsymbol{d}_{j} \quad(j=1, \ldots, 4) .
$$

The deviation vectors $\boldsymbol{d}_{1}, \boldsymbol{d}_{2}$, and $\boldsymbol{d}_{3}$ are selected from the basin calculated above. The deviation vector $\boldsymbol{d}_{4}$ is selected to confirm the validity of the aforementioned assumption that the effect of the initial current is relatively small.

We conduct a numerical simulation of 100 steps for each initial state. As a result, the gait succeeds with all the initial states. The gait continues for 100 steps without falling down, and the state approaches the fixed point. In fact, at the last step, the distance $\left\|\boldsymbol{x}_{0}-\boldsymbol{x}\right\|_{2}$, namely the distance between the fixed point and the state point at each step, becomes less than $1.0 \times 10^{-4}$ in all the cases. The angle and velocity trajectories with deviation $\boldsymbol{d}_{1}, \boldsymbol{d}_{2}, \boldsymbol{d}_{3}$, and $\boldsymbol{d}_{4}$ are shown in Figs. 9, 10, 11, and 12, respectively. In Figs. 9-11, the effect of the disturbance diminishes step by step. After several steps of
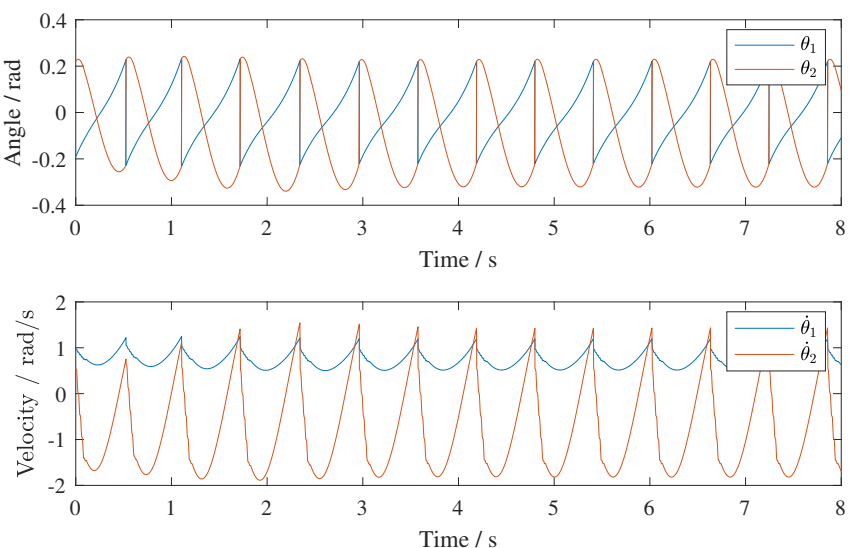

Figure 9: Angle and angular velocity trajectories with $\left(n_{1}, n_{2}\right)=(6,4)$ and with deviation $\boldsymbol{d}_{1}$.
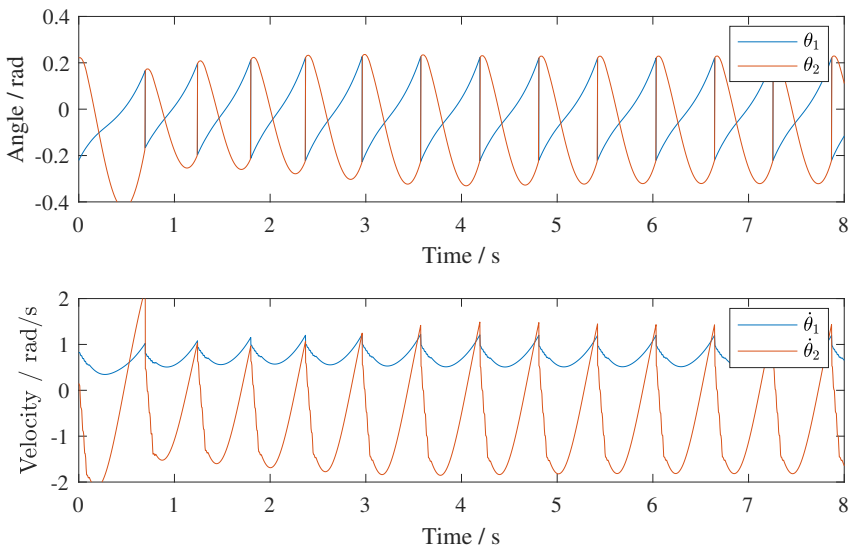

Figure 10: Angle and angular velocity trajectories with $\left(n_{1}, n_{2}\right)=(6,4)$ and with deviation $\boldsymbol{d}_{2}$.
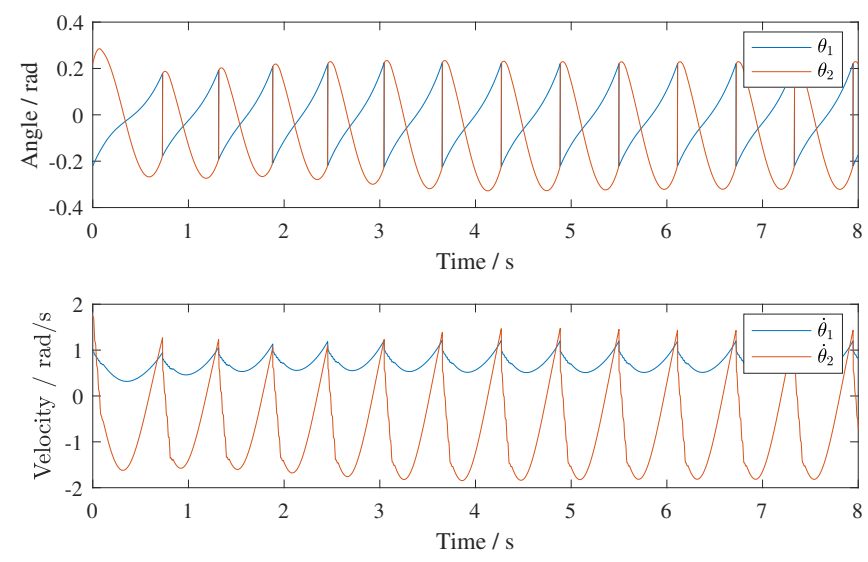

Figure 11: Angle and angular velocity trajectories with $\left(n_{1}, n_{2}\right)=(6,4)$ and with deviation $\boldsymbol{d}_{3}$. 

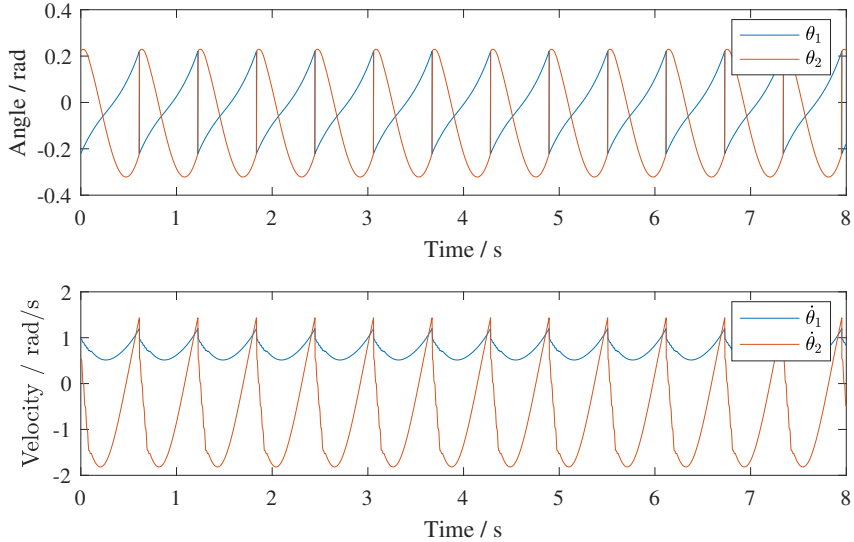

Figure 12: Angle and angular velocity trajectories with $\left(n_{1}, n_{2}\right)=(6,4)$ and with deviation $\boldsymbol{d}_{4}$.

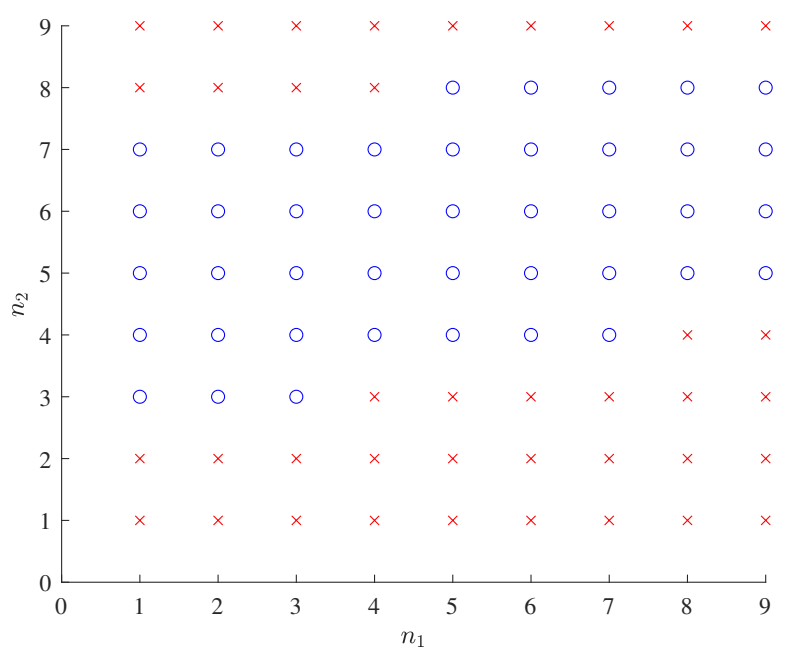

Figure 13: Combinations of $\left(n_{1}, n_{2}\right)$ which generate a successful gait. The circles "o" and crosses "x" in the plot represent the success and the failure in 100 steps gait, respectively.

transient, the angle and velocity follow almost the same trajectories as those of Fig. 5. In addition, Fig. 12 shows the gait trajectories are almost same as those of Fig. 5 from the first gait step. This indicates the validity of the aforementioned assumption that the effect of current deviation on the gait is relatively small.These results suggest the attracting property of the generated gait. This is important in practical applications; even with uncertainties such as a rough ground surface, the robot can be attracted to a stable gait.

Lastly, as an application of the attracting property, we examine the range of $\left(n_{1}, n_{2}\right)$ that generate a successful gait with a specific initial condition. Figure 13 shows the combinations of $\left(n_{1}, n_{2}\right)$ which generate a successful gait. Here a gait is defined as "successful" if it continues 100 steps starting from the initial state $\boldsymbol{x}_{0}=\overline{\boldsymbol{x}}_{(6,4)}$. The result shows that, for a single initial state, there are many candidates of the input sets that generate a successful gait. It indicates the possible application to a gait transition based on the digitized control by the power packet supply.

\section{Conclusions}

In this paper, we discussed the impulsive torque control of a biped gait with the packetized supply of power. First, we designed the packet-oriented method for supplying the impulsive torque. Second, we demonstrated the feasibility of the proposed method through numerical simulations. The successful generation of a gait was confirmed. In addition, we confirmed that the robot gait converged to the stable one even with the existence of a small disturbance of the initial condition. Finally, we observed that some properties of the gaits can be controlled by supplying a different number of power packets. The results are expected to be the basis of further research on an active control method of the gait.

Although the biped gait generation has been studied intensively for a long time, most of them are devoted to the analysis of the rich mathematical properties. The results of this paper, on the contrary, open the way to the real-world implementation of the concept. Moreover, they give some clues for a novel approach in the field of motion control. For example, the torque (current) input waveforms in the numerical simulation seem similar to a rhythmic burst of neuron models [38]. Here arises the possibility to replace a CPG network, which has been defined in the cyber world, with a network based on a flow of a physical quantity. This perspective offers a way of the integration of information and physical quantities, which is essential in cyber-physical systems [39].

\section{Acknowledgements}

This work was partially supported by JSPS KAKENHI grant number JP18J11530 and by Program on Open Innovation Platform with Enterprises, Research Institute and Academia (OPERA) from Japan Science and Technology Agency.

\section{Conflict of interest}

The authors declare that they have no conflict of interest.

\section{References}

[1] McGeer, T.: Passive Dynamic Walking. The International Journal of Robotics Research 9(2), 62-82 (1990). DOI 10.1177/027836499000900206

[2] Goswami, A., Thuilot, B., Espiau, B.: CompassLike Biped Robot Part I: Stability and Bifurcation 
of Passive Gaits. Research Report RR-2996, INRIA (1996)

[3] Garcia, M., Chatterjee, A., Ruina, A., Coleman, M.: The Simplest Walking Model: Stability, Complexity, and Scaling. Journal of Biomechanical Engineering 120(2), 281-288 (1998). DOI 10.1115/1.2798313

[4] Tavakoli, A., Hurmuzlu, Y.: Robotic locomotion of three generations of a family tree of dynamical systems. Part I: Passive gait patterns. Nonlinear Dynamics 73(3), 1969-1989 (2013). DOI 10.1007/s11071-013-0918-4

[5] Guckenheimer, J., Holmes, P.: Nonlinear Oscillations, Dynamical Systems, and Bifurcations of Vector Fields, vol. 42. Springer-Verlag New York, New York, NY (1983). DOI 10.1007/978-1-4612-1140-2

[6] Znegui, W., Gritli, H., Belghith, S.: Design of an explicit expression of the Poincaré map for the passive dynamic walking of the compass-gait biped model. Chaos, Solitons \& Fractals 130, 109436 (2020). DOI 10.1016/j.chaos.2019.109436

[7] Wang, Y., Cao, H., Jiang, J.: An Improved Method for Estimating the Domain of Attraction of Passive Biped Walker (2019). DOI 10.1155/2019/2868543

[8] Obayashi, I., Aoi, S., Tsuchiya, K., Kokubu, H.: Common formation mechanism of basin of attraction for bipedal walking models by saddle hyperbolicity and hybrid dynamics. Japan Journal of Industrial and Applied Mathematics 32(2), 315-332 (2015). DOI 10.1007/s13160-015-0181-9

[9] Obayashi, I., Aoi, S., Tsuchiya, K., Kokubu, H.: Formation mechanism of a basin of attraction for passive dynamic walking induced by intrinsic hyperbolicity. Proceedings of the Royal Society A: Mathematical, Physical and Engineering Sciences 472(2190), 20160028 (2016). DOI 10.1098/rspa. 2016.0028

[10] Okamoto, K., Aoi, S., Obayashi, I., Kokubu, H., Senda, K., Tsuchiya, K.: Fractal mechanism of basin of attraction in passive dynamic walking. Bioinspiration \& Biomimetics (2020). DOI 10.1088/ 1748-3190/ab9283

[11] Goswami, A., Espiau, B., Keramane, A.: Limit Cycles in a Passive Compass Gait Biped and PassivityMimicking Control Laws. Autonomous Robots 4(3), 273-286 (1997). DOI 10.1023/A:1008844026298

[12] Asano, F., Luo, Z.W., Yamakita, M.: Biped gait generation and control based on a unified property of passive dynamic walking. IEEE Transactions on Robotics 21(4), 754-762 (2005). DOI 10.1109/TRO. 2005.847610
[13] Spong, M.W., Holm, J.K., Lee, D.: Passivity-Based Control of Bipedal Locomotion. IEEE Robotics \& Automation Magazine 14(2), 30-40 (2007). DOI 10.1109/MRA.2007.380638

[14] Sinnet, R.W., Ames, A.D.: Energy shaping of hybrid systems via control Lyapunov functions. In: 2015 American Control Conference (ACC), pp. 5992-5997 (2015). DOI 10.1109/ACC.2015.7172280

[15] de-León-Gómez, V., Santibañez, V., Sandoval, J.: Interconnection and damping assignment passivitybased control for a compass-like biped robot. International Journal of Advanced Robotic Systems 14(4), 1729881417716593 (2017). DOI 10.1177/ 1729881417716593

[16] Yeatman, M., Lv, G., Gregg, R.D.: Decentralized Passivity-Based Control With a Generalized Energy Storage Function for Robust Biped Locomotion. Journal of Dynamic Systems, Measurement, and Control 141(10) (2019). DOI 10.1115/1.4043801

[17] Fu, C., Tan, F., Chen, K.: A simple walking strategy for biped walking based on an intermittent sinusoidal oscillator. Robotica 28(6), 869-884 (2010). DOI $10.1017 /$ S0263574709990713

[18] Fu, C., Wang, J., Chen, K., Yu, Z., Huang, Q.: A walking control strategy combining global sensory reflex and leg synchronization. Robotica 34(5), 973994 (2016). DOI 10.1017/S0263574714002008

[19] Gritli, H., Belghith, S., Khraief, N.: OGY-based control of chaos in semi-passive dynamic walking of a torso-driven biped robot. Nonlinear Dynamics 79(2), 1363-1384 (2015). DOI 10.1007/ s11071-014-1747-9

[20] Gritli, H., Belghith, S.: Walking dynamics of the passive compass-gait model under OGY-based control: Emergence of bifurcations and chaos. Communications in Nonlinear Science and Numerical Simulation 47, 308-327 (2017). DOI 10.1016/j.cnsns. 2016.11.022

[21] Kuo, A.D.: Energetics of Actively Powered Locomotion Using the Simplest Walking Model. Journal of Biomechanical Engineering 124(1), 113 (2001). DOI 10.1115/1.1427703

[22] Moon, J.S., Stipanović, D.M., Spong, M.W.: Gait Generation and Stabilization for Nearly Passive Dynamic Walking Using Auto-distributed Impulses. Asian Journal of Control 18(4), 1343-1358 (2016). DOI 10.1002/asjc.1206

[23] Mochiyama, S., Hikihara, T.: Multiple Gaits of Biped Robot with Impulsive Excitation by Power 
Packets. In: 2019 International Symposium on Nonlinear Theory and Its Applications, pp. 381-384. Kuala Lumpur, Malaysia (2019)

[24] Takuno, T., Koyama, M., Hikihara, T.: In-home Power Distribution Systems by Circuit Switching and Power Packet Dispatching. In: Proceedings of the 1st IEEE International Conference on Smart Grid Communications, pp. 427-430. Gaithersburg, MD, USA (2010). DOI 10.1109/SMARTGRID. 2010.5622079

[25] Takahashi, R., Azuma, S.i., Hasegawa, M., Ando, H., Hikihara, T.: Power Processing for Advanced Power Distribution and Control. IEICE Transactions on Communications E100.B(6), 941-947 (2017). DOI 10.1587/transcom.2016EBN0005

[26] Gelenbe, E.: Energy Packet Networks: Adaptive Energy Management for the Cloud. In: Proceedings of the 2nd International Workshop on Cloud Computing Platforms, 1, pp. 1-5. Bern, Switzerland (2012). DOI 10.1145/2168697.2168698

[27] Gelenbe, E., Abdelrahman, O.H.: An Energy Packet Network model for mobile networks with energy harvesting. Nonlinear Theory and Its Applications, IEICE 9(3), 322-336 (2018). DOI $10.1587 /$ nolta. 9.322

[28] Sugiyama, H., Chatani, M., Simizu, R., Yasui, K.: Pulsed power network with inherent operating procedure and multiple relaying of power routers. In: Proceedings of 2017 IEEE 6th Global Conference on Consumer Electronics, pp. 1-2. Nagoya, Japan (2017). DOI 10.1109/GCCE.2017.8229468

[29] Takahashi, R., Azuma, S.i., Hikihara, T.: Power Regulation with Predictive Dynamic Quantizer in Power Packet Dispatching System. IEEE Transactions on Industrial Electronics 63(12), 7653-7661 (2016). DOI 10.1109/TIE.2016.2591898

[30] Mochiyama, S., Hikihara, T.: Packet-based feedback control of electrical drive and its application to trajectory tracking of manipulator. International Journal of Circuit Theory and Applications 47(4), 612-632 (2019). DOI 10.1002/cta.2603

[31] Kimoto, T.: Material science and device physics in $\mathrm{SiC}$ technology for high-voltage power devices. Japanese Journal of Applied Physics 54(4), 1-24 (2015). DOI 10.7567/JJAP.54.040103

[32] Collins, S., Ruina, A., Tedrake, R., Wisse, M.: Efficient bipedal robots based on passive-dynamic walkers. Science 307(5712), 1082-1085 (2005). DOI 10.1126/science.1107799
[33] Moon, J.S., Bae, J.: Gait optimization and energetics of ballistic walking for an underactuated biped with knees. Nonlinear Dynamics 85(3), 1533-1546 (2016). DOI 10.1007/s11071-016-2777-2

[34] Craig, J.J.: Introduction to Robotics: Mechanics and Control, second edn. Addison-Wesley (1989)

[35] MAXON: MAXON RE40 148877. https: //www.maxongroup.com/maxon/view/product/ motor/dcmotor/re/re40/148877

[36] Chicone, C.: Ordinary Differential Equations with Applications, second edn. Texts in Applied Mathematics. Springer-Verlag, New York (2006). DOI 10.1007/0-387-35794-7

[37] Gritli, H., Khraief, N., Belghith, S.: Chaos control in passive walking dynamics of a compassgait model. Communications in Nonlinear Science and Numerical Simulation 18(8), 2048-2065 (2013). DOI 10.1016/j.cnsns.2012.12.009

[38] Ghigliazza, R.M., Holmes, P.: A Minimal Model of a Central Pattern Generator and Motoneurons for Insect Locomotion. SIAM Journal on Applied Dynamical Systems 3(4), 671-700 (2004). DOI 10. $1137 / 040607563$

[39] Kim, K.D., Kumar, P.R.: Cyber-Physical Systems: A Perspective at the Centennial. Proceedings of the IEEE 100(Special Centennial Issue), 1287-1308 (2012). DOI 10.1109/JPROC.2012.2189792 